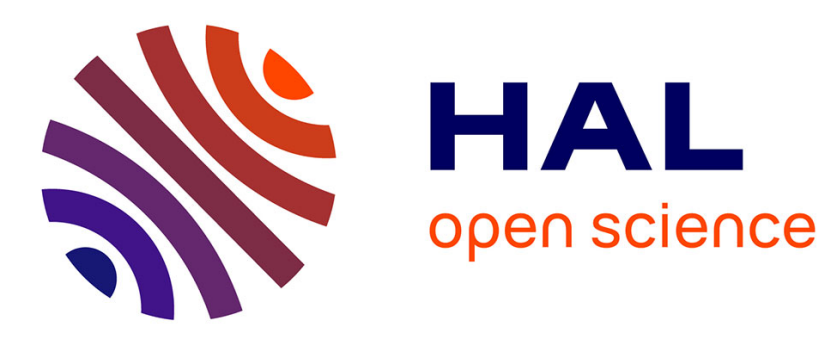

\title{
L'oeuvre scientifique des premiers professeurs de la Faculté de Pharmacie de Lyon
}

\author{
Cécile Maître, Philippe Jaussaud
}

\section{To cite this version:}

Cécile Maître, Philippe Jaussaud. L'oeuvre scientifique des premiers professeurs de la Faculté de Pharmacie de Lyon. Bulletin d'histoire et d'épistémologie des sciences de la vie , 2014, 21 (2), pp.131148. halshs-01085869

\section{HAL Id: halshs-01085869 \\ https://shs.hal.science/halshs-01085869}

Submitted on 21 Nov 2014

HAL is a multi-disciplinary open access archive for the deposit and dissemination of scientific research documents, whether they are published or not. The documents may come from teaching and research institutions in France or abroad, or from public or private research centers.
L'archive ouverte pluridisciplinaire HAL, est destinée au dépôt et à la diffusion de documents scientifiques de niveau recherche, publiés ou non, émanant des établissements d'enseignement et de recherche français ou étrangers, des laboratoires publics ou privés. 


\title{
L'œuvre scientifique des premiers professeurs de la Faculté de Pharmacie de Lyon.
}

\author{
Cécile MAÎTRE et Philippe JAUSSAUD*
}

RÉSUMÉ - Une faculté mixte de médecine et pharmacie est créée à Lyon en 1874. Ses premiers professeurs chargés d'enseigner les sciences pharmaceutiques sont très actifs au plan de la recherche. Outre les sciences physiques, leurs travaux concernent aussi bien le domaine de l'histoire naturelle botanique, zoologie - que dans celui des sciences biomédicales ou agronomiques - matière médicale, biochimie, toxicologie, phytopharmacie, hygiène. Les résultats obtenus sont d'une grande importance pour la société : l'anatomie végétale permet de détecter des falsifications de plantes médicinales; des méthodes de lutte contre le Phylloxéra sont mises au point ; l'hématologie médico-légale se développe, offrant de nouveaux moyens d'expertises judiciaires ; des études toxicologiques - concernant le monoxyde de carbone ou des colorants alimentaires - permettent d'améliorer l'hygiène et de protéger la santé publique : sont étudiés. La biochimie fondamentale se développe, notamment dans le domaine des protéines, et les résultats obtenus en endocrinologie annoncent la découverte de l'insuline. A contrario, les travaux de pharmacologie pure sont rares. Toutes ces recherches ont des conséquences pédagogiques, comme l'édition de manuels, l'installation d'un jardin botanique ouvert aux étudiants ou la diffusion des connaissances vers le grand public.

MOTS-CLEFS : faculté, pharmacie, Lyon, professeurs, travaux.

\begin{abstract}
A double faculty of medicine and pharmacy is founded in Lyon, in 1874. Its first professors in charge of learning the pharmaceutical sciences are very active scientists. Besides the physical sciences, their works concern no less the field of the natural history - botany, zoology -, than the field of biomédical or agronomical sciences - médical material, biochemistry, toxicology, phytopharmacy, hygiène. The results obtained are of grêât importance for the society : the végétal anatomy permits to detect adultération of médicinal plants ; fighting méthodes against Phylloxera are perfected; the forensic haematology is greatly improved, giving to the justice new means for court-ordered appraisal ; toxicological studies - upon carbon monoxide or food dyes - permit to improve hygiene and protect the public health.The fundamental biochemistry makes progress, especially in the field of proteins, and the results obtained in endocrinology announce the discovery of insulin. A contrario, works are rare in the field of pure pharmacology. All these works take on pedagogical conséquences, as handbooks publication, planting a botanical garden for students or spreading the knowledge towards the général public.
\end{abstract}

KEYWORDS : faculty, pharmacy, Lyon, professors, works.

* EA 4148 S2HEP (Sciences et Société : Historicité, Éducation, Pratiques), Université Claude Bernard Lyon 1, Domaine Scientifique de La Doua, Bâtiment La Pagode, 38 Bd Niels Bohr, 69622 VILLEUBANNE CEDEX. 


\section{Introduction}

À Lyon, l'enseignement de la pharmacie s'est trouvé longtemps associé à celui de la médecine. Car c'est une faculté mixte qui remplace, en 1874, l'ancienne école préparatoire de médecine et de pharmacie de la ville ${ }^{1}$. Il faudra attendre la fin de l'année 1968, pour qu'une Unité d'Enseignement et de Recherche (UER) de pharmacie pure soit constituée. Quoi qu'il en soit, la création de la faculté mixte de Lyon permet d'accueillir un flux croissant d'étudiants et rend possible l'acquisition sur place du grade de docteur. L'inauguration de la nouvelle institution, ainsi que la première rentrée universitaire, ont lieu en 1877. Cinq ans plus tard, la faculté mixte se trouvera transférée dans le palais universitaire édifié par l'architecte Abraham Hirsch (18281913) au bord du Rhône. L'installation des laboratoires, achevée seulement en 1883, sera suivie de travaux d'agrandissement au cours de la décennie 1890-1900².

Les enseignants de l'ancienne école préparatoire - pharmaciens ou médecins - constituent le noyau du corps professoral de la faculté mixte, laquelle se trouve initialement dotée de trentecinq chaires magistrales ${ }^{3}$. Vingt-cinq professeurs titulaires et vingt-deux agrégés ont la charge d'environ trois cents étudiants. Plusieurs enseignements sont mixtes, regroupant les futurs médecins et pharmaciens. Mais, quatre chaires concernent plus spécifiquement le cursus de pharmacie :

- la chaire de «Chimie médicale et pharmaceutique » - future chaire de « Chimie biologique et médicale »- est occupée successivement par Alexandre Glénard (de 1877 à 1889) et Louis Hugounenq (de1891 à 1930).

- la chaire de «Pharmacie », puis de «Pharmacie et pharmacologie » échoit à Ferdinand Crolas (de 1879 à 1903), puis à Albert Florence (de 1903 à 1922).

- la chaire de « Matière médicale » - qui sera transformée en « Matière médicale et botanique » est successivement dirigée par Désiré Cauvet (de 1877 à 1890), Albert Florence (de 1891 à 1903), Georges Eugène Charles Beauvisage (de 1903 à 1912), Barthélémy Moreau (de 1912 à 1922) et Philippe Marie Bretin (de 1923 à 1931).

- la chaire de «Chimie organique et toxicologie». Créée à l'intention de Paul Cazeneuve (de 1881 à1909) après partition de la chaire de « Médecine légale et toxicologie », elle échoit ensuite à Albert Morel (de1909 à 1943) .

Toutes ces chaires sont occupées par des universitaires nantis du diplôme de pharmacien, la plupart étant de surcroît docteurs en médecine.

L'œuvre scientifique des premiers professeurs de pharmacie de la faculté mixte de Lyon est mal connue. De fait, elle est peu évoquée, voire carrément passée sous silence, dans les publications commémoratives ayant trait à la vie institutionnelle lyonnaise ${ }^{5}$. L'homme de science

\footnotetext{
1 Laquelle remplace, depuis 1841, l'ancienne école secondaire de médecine, cf. Despierres G. (1987) «L'École secondaire de Médecine (1821-1841), in : Bouchet A. La médecine à Lyon - Des origines à nos jours, Lyon, Hervas, pp. 92-95 et Despierres G. (1987) «L'École préparatoire de Médecine et de Pharmacie », Ibid, pp. 96 -100.

2 Despierres G. (1987) «La Faculté mixte de Médecine et de Pharmacie et son éclatement», in: Bouchet A. La médecine à Lyon - Des origines à nos jours, Lyon, Hervas, pp. 101- 116 et Cazeneuve P. (1920) Sur les origines de la faculté de médecine et de pharmacie de Lyon, Lyon, Association typographique.

${ }^{3}$ Guiart J. (1941) L'école médicale lyonnaise : catalogue commenté de la section régionale du musée historique de la faculté mixte de médecine et pharmacie de Lyon, Paris, Masson, pp. 106-107.

${ }^{4}$ De nature essentiellement chimique, les travaux d'Albert Morel, ne seront pas envisagés ici.

${ }^{5}$ Cf., par exemple : Drevon B. (1977) «La pharmacie dans la faculté mixte de médecine et pharmacie de Lyon », in : Le Centenaire de la faculté mixte de médecine et de pharmacie de Lyon, Cahiers de médecine, vol. 2, n³6, pp. 2067-2069.
} 
s'estompe même parfois, dans les mémoires, au bénéfice de l'homme politique : les noms de Beauvisage et de Cazeneuve, tous deux professeurs de la faculté mixte, auraient-ils été choisis pour baptiser des rues de Lyon s'il n'avaient été des élus municipaux et parlementaires ?

Dans ce contexte, il apparaît utile de présenter les grandes lignes de l'oeuvre scientifique des premiers pharmaciens en charge de l'enseignement de la pharmacie à l'université de Lyon ${ }^{6}$. Afin d'éviter les écueils liés au traitement d'un sujet trop vaste, les sciences physico-chimiques ne seront pas envisagées ici ${ }^{7}$. Seuls seront décrits les travaux relevant des sciences de la vie et de la santé : botanique et matière médicale, zoologie et phytopharmacie $^{8}$, biologie médicale, pharmacologie et toxicologie.

\section{1) - Botanique et matière médicale}

Les travaux des professeurs de botanique de la faculté mixte s'inscrivent dans un cadre disciplinaire construit et illustré en France, entre la fin du XIXème et le début du XXème siècle, par des savants comme Philippe van Tieghem (1839-1914) au Muséum de Paris ou Gaston Bonnier à la Sorbonne (1853-1922). Dans les domaines plus spécifiques de la botanique médicale et de la pharmacognosie, l'influence de plusieurs professeurs de la faculté de pharmacie de Paris tels Léon Guignard (1852-1928), Gustave Planchon (1833-1900) ou Émile Perrot (1867-1951) ${ }^{9}$ s'est très certainement exercée. L'activité des universitaires lyonnais se rattache à quatre champs de recherche principaux : l'anatomie et la biologie végétales, ainsi que la matière médicale.

\section{Anatomie végétale}

Les recherches anatomiques de Désiré Cauvet (1827-1890) se rattachent à de nombreuses familles. Il consacre sa thèse d'agrégation à la morphologie des Solanées, puis étudie l'organographie des Cactées, du Tamier commun et de la Salsepareille, la vrille des Ampélidées et des Cucurbitacées, le bourgeon et les stipules des Monocotylédones, les caractères distinctifs des Rhubarbes, etc. Dans le domaine de l'histologie végétale, Cauvet s'intéresse à la structure des cladodes du Fragon faux houx, de la racine du Ciste et du ricin d'Algérie, de même qu'à « la constitution histologique des Ipécacuanhas». Il publie une note sur la «Kernscheide» - un

\footnotetext{
${ }^{6}$ Cf. Maître C. (2013) Les premiers professeurs de la faculté de pharmacie de Lyon, Thèse de Doctorat d'État en Pharmacie, 15 janvier 2013, Lyon, Université Claude Bernard Lyon 1, 118 p.

7 Pour les enseignants dont une partie de la carrière s'est déroulée ailleurs qu'à Lyon, nous n'opérerons pas de distinction entre les travaux réalisés à la faculté mixte et les autres. Désiré Cauvet, par exemple, a initialement professé à Strasbourg et à Nancy. Nombre de ses collègues passent l'agrégation à Paris avant de rejoindre Lyon.

${ }^{8}$ La botanique et la zoologie se trouvent à la base de deux sciences pharmaceutiques : la matière médicale ou pharmacognosie - ancienne « histoire naturelle des médicaments » - et la phytopharmacie. D’où les regroupements disciplinaires opérés ici, qui peuvent d'ailleurs s'effectuer au cours de l'évolution des structures institutionnelles : la chaire de «Matière médicale » de la faculté mixte, par exemple, est rebaptisée « Matière médicale et Botanique » pour Beauvisage.

${ }^{9}$ Cf. Paris, R. (1954) «Botanique médicale», in : Davy de Virville, A., Histoire de la botanique en France, Paris, Société d'Édition d'Enseignement Supérieur, p. 305.
} 
manchon cellulaire présent dans les racines des Angiospermes - et conduit des recherches histochimiques sur le Cytinet, un parasite du Ciste ${ }^{10}$.

Georges Beauvisage (1852-1925) considère divers organes végétaux, sous les angles de l'anatomie normale, de l'anatomie pathologique ou de la tératologie. Il conduit des études organographiques visant à rationnaliser et simplifier la classification des fruits et des inflorescences. Contestant les observations de Karl von Nägeli (1817-1891) sur les faisceaux libéro-ligneux de la tige de l'Igname, Beauvisage précise le nombre et le trajet de ces formations. Le pharmacien conduit une étude semblable sur la racine de Belladone, mettant en évidence l'enclavement de tubes criblés dans le xylème. Par ailleurs, Beauvisage démontre l'existence constante - bien que parfois discrète - de bractées dans la famille des Crucifères.

S'appuyant au besoin sur des observations microscopiques, Beauvisage décrit chez les plantes des anomalies ou des formations atypiques : des roses «prolifères ${ }^{11}$, des feuilles de Haricot dotées d'un nombre inhabituel de folioles, des canaux sécréteurs oblitérés à divers degrés chez le Pin sylvestre ou une formation subéreuse cicatricielle chez l'Iris.

Élève de Beauvisage, Bretin (1874-1931) consacre de nombreuses investigations à la «botanique morphologique et anatomique ${ }^{12}$ des plantes médicinales ou toxiques. Il étudie en particulier une variété indigène d'Artemisia, ressemblant au «semen contra - exotique et vermifuge -, ainsi que les fleurs de l'aubépine vulgaire (Crataegus oxyacantha) et la feuille de Coca (Erythroxylon coca). Sur cette dernière, grâce à des coupes transversales sériées, Bretin montre que les deux lignes courbes délimitant l' «area » résultent du mode d'enroulement des folioles dans le bourgeon ${ }^{13}$. En lien avec leur toxicité, Bretin s'attache à la détermination anatomique des fruits indigènes et des baies de Solanacées. Cette dernière famille bénéficie d'une notable faveur auprès des professeurs de pharmacie de la faculté mixte. Il est vrai que le sujet est classique : il se trouve déjà abordé, vingt ans plus tôt, dans le mémoire d'agrégation d'Alphonse Milne-Edwards ${ }^{14}$.

\section{Biologie végétale}

Divers phénomènes biologiques retiennent l'attention de Cauvet, comme l'absorption des liquides colorés par les racines, le mode d'ascension de la sève ou l'excrétion des matières non assimilables. Le pharmacien s'intéresse également au parasitisme des racines de Cistes par le

\footnotetext{
${ }^{10}$ Anonyme (1890) "Mr. Le Professeur Cauvet", L'Union Pharmaceutique, 31ème année, pp. 78-80 et LABRUDE P. (2009) "Nouvelles recherches sur Philippe Désiré Cauvet (Agde 1827-Lyon 1890), pharmacien militaire, naturaliste, agrégé et professeur, Revue d'Histoire de la Pharmacie, n³64, pp. 392-393.

${ }^{11}$ Une fleur est dite "prolifère" lorsque d'autres fleurs naissent en son centre.

${ }^{12}$ Manceau P. (1931) "Le professeur Philippe Bretin (1874-1931)”, Lyon Pharmaceutique, p. 57

${ }^{13}$ L'area" est une zone elliptique qui constitue un critère d'identification de la plante. Pour élucider son origine, Bretin suit le développement d'un bourgeon de Coca provenant du Parc de la Tête d'Or à Lyon. Cf. Moreau B. (1922) Rapport sur la candidature de Monsieur le Docteur Bretin, Agrégé, Chargé de Cours, à la chaire de Matière médicale et Botanique de la Faculté de Médecine et Pharmacie de Lyon. Document manuscrit. Dossier personnel de Philippe Bretin, ISPB - Faculté de Pharmacie de Lyon, Université Claude Bernard Lyon 1, p. 9.

${ }^{14}$ Cf. Milne-Edwards A. (1864) De la famille des Solanacées, Paris, E. Martinet, 137 p. Malgré la nature botanique de ce sujet, Milne-Edwards sera professeur de zoologie au Muséum et à l'École supérieure de pharmacie de Paris.
} 
Cytinet ${ }^{15}$. L'étude de son Cours élémentaire de botanique, publié en 1879 et réédité en 1885, fait ranger Cauvet parmi les premiers propagateurs du darwinisme en France ${ }^{16}$.

Bretin étudie l'action sur l'Aucuba japonica du froid, lequel produit une décoloration des feuilles de la plante. Il s'intéresse également au rôle des insectes dans la pollinisation, effectuant des observations sur diverses espèces végétales, comme les Lamium, les Orchis ou les Arum.

\section{Matière médicale}

Pour fournir des éléments de diagnose différentielle, Cauvet publie trois mémoires, relatifs respectivement à la structure histologique des racines du Grenadier, du Vérâtre et des Salsepareilles ${ }^{17}$.

Dans sa thèse de doctorat en médecine, intitulée Contribution aux origines botaniques de la Gutta-Percha (1881), Beauvisage s'attache à identifier les diverses espèces d'arbres fournissant la drogue. La tâche est ardue, en raison du caractère contradictoire des observations réalisées et de «l'absence à peu près totale d'échantillons botaniques complets ${ }^{18}$. Peu après, pour sa thèse d'agrégation, Beauvisage conduit des investigations sur les galles d'intérêt médical ou industrie ${ }^{19}$. Ayant caractérisé la présence d'inuline dans le faux Ipécacuanha blanc du Brésil, il réalise une étude histologique complète de la racine de cette plante - incluant l'observation de cristaux. Les études originales de Beauvisage sur les «bois pharaoniques » des cercueils royaux de l'ancienne Égypte, l'if et l'ébène, établissent un lien entre la matière médicale et l'archéologie

Bretin réalise lui-même ou inspire à ses élèves plusieurs travaux de matière médicale «comportant à la fois des études botaniques, micrographiques, chimiques et pharmacologiques $»^{20}$. Des recherches sur le chanvre indien montrent que le Cannabis sativa pousse facilement en France et produit des inflorescences très riches en résine. Bretin s'attache aux diagnoses différentielles et à la recherche, dans les drogues, d'impuretés ou de falsifications. Ainsi met-il en évidence les caractères morphologiques permettant de distinguer la mauve officinale d'une fausse mauve d'Algérie, le Juniperus thurifera de la Sabine ou l'Hysope de la Sarriette. Il s'intéresse aux falsifications des feuilles d'Hamamelis virginica et de l'Adonis vernalis. Á ce dernier se trouvent fréquemment substituées d'autres espèces d'Adonis, comme le constate Bretin qui pousse ses investigations jusqu'au domaine de la phytochimie : une comparaison des teneurs en principes actifs de divers Adonis l'amène à conclure qu'A. vernalis doit rester le seul officinal ${ }^{21}$.

Ses compétences pratiques conduisent Bretin à présider le Comité régional lyonnais des plantes médicinales. Il s'agit d'une branche du Comité interministériel des plantes médicinales et des plantes à essences, dépendant de l'Office national des matières premières pour la droguerie, la pharmacie, la parfumerie et la distillerie. Dans ce cadre, Bretin dirige au laboratoire de matière

\footnotetext{
15 Anonyme (1890), pp. 79-80.

${ }^{16}$ Bange C (2013) Le botaniste Désiré Cauvet et l'enseignement des théories transformistes à la Faculté de médecine et de pharmacie de Lyon à la fin du XIXème siècle, Bulletin Mensuel de la Société Linnéenne de Lyon, Vol. 82, fasc. 1-2, pp.

17 Anonyme (1890), pp. 78-80.

18 Beauvisage G. (1891) Exposé des titres et travaux scientifiques, Lyon, Association typographique, p. 13.

${ }^{19}$ Beauvisage G. (1883) Les galles utiles, Paris, Doin, 99 p.

${ }^{20}$ Moreau B. (1922), Op. cit., p. 8.

${ }^{21}$ Bretin découvre aussi des fraudes dans des échantillons commerciaux d'Adonis vernalis : il signale l'incorporation de fleurs de renoncule ou de tiges de prêles finement découpées, Cf. Moreau B. (1922), Op. cit., p. 10.
} 
médicale de la faculté mixte un service spécial doté de plusieurs missions : donner gratuitement des renseignements sur la culture, la dessiccation et la conservation des plantes médicinales, ainsi que sur l'identité et la pureté des drogues, inventorier et valoriser les ressources locales et mettre en relation les récoltants et les acheteurs.

Pharmacien militaire au début de sa carrière ${ }^{22}$, Camille Victor Théophile Sambuc (185919?) profite de ses affectations à Pondichéry, au Sénégal et à la Guadeloupe pour étudier les flores tropicales et réaliser des observations phytogéographiques ${ }^{23}$. Sa thèse de pharmacie est consacrée à l'étude des plantes de Sénégambie auxquelles les populations locales attribuent des propriétés thérapeutiques. Il s'agit notamment de plantes amères fébrifuges appartenant à diverses familles - Rubiacée, Ménispermacée, Légumineuse - et d'un acacia taenifuge ${ }^{24}$.

Ferdinand Gabriel Crolas (1841-1903), qui occupe la chaire de « Pharmacie », contribue à la matière médicale seulement par le biais d'investigations phytochimiques et officinales : «ses procédés de dosage ingénieux de la morphine dans l'opium et de la quinine dans les quinquinas, son procédé d'utilisation des poussières de thé pour l'extraction de la caféine, la préparation d'extraits non résineux et complètement solubles dans l'eau, de quinquina et de kola ${ }^{25}$. De même Jean-Baptiste Paul Cazeneuve (1852-1934), futur professeur de "Chimie organique et Toxicologie », soutient en 1875 sa thèse de pharmacie sur la Recherche et extraction des alcaloïdes $^{26}$. Son collègue Barthélémy Moreau (1866-1923), qui est professeur de «Matière médicale et Botanique » (1912) avant d'occuper la chaire de «Pharmacie » (1922), s'intéresse au chanvre indien ${ }^{27}$. Il dirige également des thèses de doctorat en pharmacie relatives au dosage de certains alcaloïdes dans les drogues : la morphine dans l'opium et les alcaloïdes totaux dans l'écorce de quinquina ${ }^{28}$. De tels travaux rappellent, bien sûr, des études de Crolas qui viennent d'être citées.

La thèse d'agrégation d'Albert Florence (1851-1927) - nommé successivement professeur de «Matière médicale et botanique », puis de «Pharmacie »- porte sur Les alcalö̈des des Solanées (1886). L'importance académique de cette famille végétale a été précédemment soulignée. Enfin, Louis Hugounenq (1860-1942), professeur de «Chimie médicale et pharmaceutique », puis de «Chimie biologique et médicale ${ }^{29}$, étudie avec Paul Cazeneuve (1852-1934) deux constituants du bois de Santal rouge (Pterocarpus santalinus) : la ptérocarpine et l'homoptérocarpine ${ }^{30}$.

\footnotetext{
${ }^{22}$ C'est-à-dire de 1881 à 1888. Pharmacien et médecin, Sambuc est agrégé de chimie de la faculté mixte de Lyon en 1898. D'abord chargé de cours, il est nommé chef des travaux pratiques de chimie en 1910. Il rejoint en 1912 la faculté mixte d'Alger où il achèvera sa carrière comme professeur de chimie biologique. Cf. Sambuc C. (1913) Titres et services du Dr Camille Sambuc, Lyon, Rey, 29 p.

${ }^{23}$ Il s'intéresse aussi à la météorologie et à la physique du globe : sa thèse de médecine rapporte des Recherches sur le climat d'Alger (1897).

${ }^{24}$ Sambuc C. (1913) Op. cit., p. 11-12.

${ }^{25}$ Moreau B. (1903) "Le professeur Crolas”, Bulletin des Sciences Pharmacologiques, T. 8, p. 91.

${ }^{26}$ Chambon M. (1934) "Le professeur Paul Cazeneuve (1852-1934)", Bulletin des Sciences Pharmacologiques, T. $41, n^{\circ} 6$, p. 357.

${ }^{27}$ Il conduit également, avec Bietrix, une étude sur la falsification de l'huile de foie de morue

${ }^{28}$ Bretin P. (1924) "Notice biographique. Le Professeur Moreau, Bulletin des Sciences Pharmacologiques, T. 31, $\mathrm{n}^{\circ} 2$, p. 110.

${ }^{29}$ C'est lui qui fera transformer l'intitulé de sa chaire. Cf. Weitz R. (1942) "Le doyen honoraire Louis-Joseph Hugounenq (1860-1942)", Bulletin des Sciences Pharmacologiques, vol. 49, n 10-11-12, p. 91.

${ }^{30}$ Weitz R. "Le doyen honoraire Louis-Joseph Hugounenq (1860-1942)", Bulletin des Sciences Pharmacologiques, vol. $49, \mathrm{n}^{\circ} 10-11-12$, p. 91.
} 


\section{2) - Parasitologie et phytopharmacie}

Dans ce domaine, hormis de courtes notes publiées par Cauvet sur des Cestodes parasites de l'Homme - Ténia et Bothriocéphale -, ${ }^{31}$ les travaux des professeurs de la faculté mixte concernent la lutte contre des phytoparasites de la vigne.

Bretin publie ainsi des «considérations sur le mode de culture des pyrèthres et leur utilisation comme insecticides de la vigne, soit à l'état de poudre, soit en extrayant le principe actif et en l'incorporant à une solution savonneuse. On substituerait ainsi une poudre inoffensive pour l'Homme et les animaux supérieurs à des produits chimiques dangereux ou toxiques, soit par eux-mêmes, soit par leurs impuretés $»^{32}$.

Toujours dans le domaine phytopharmaceutique, Crolas s'investit considérablement sur le terrain. En 1875, alors que sa carrière universitaire débute, le ministère de l'agriculture le charge de développer une lutte scientifique contre le Phylloxera (Daktulosphaira vitifoliae) ${ }^{33}$. Crolas poursuivra cette mission pendant plus de vingt ans. "Il organisa dans diverses régions des champs d'expériences, où il essaya méthodiquement les divers traitements et les divers insecticides qui lui étaient adressés de France et de l'étranger (...). Il eut à lutter contre l'hostilité des propriétaires, contre le mauvais vouloir des vignerons, contre les cabales organisées par des paysans, l'accusant d'empoisonner le terrain et de le rendre stérile ${ }^{34} \gg$.

\section{3) - Biologie médicale}

Les travaux de biologie médicale des pharmaciens de la faculté mixte peuvent être décrits selon quatre grands thèmes de recherche : le sang et ses constituants, la régulation de la glycémie, l'hématologie médico-légale et les biomolécules azotées.

Relevant du premier thème, des Recherches sur l'hématine (1875) permettent à Cazeneuve d'obtenir son doctorat en médecine ${ }^{35}$. Moreau réalise, dans le même contexte, une Recherche sur le dosage du fer dans le sang et sur la teneur en fer du sang du nouveau-né (1901). Ce travail de chimie biologique valide, chez l'Homme, une hypothèse émise par Gustav von Bunge (1844-1920) et reprise par Louis Marie Joseph Hugounenq (1860-1942) : le lait étant très pauvre en fer, le jeune devrait disposer en naissant une « provision de ce métal pour l'utiliser, au fur et à mesure de ses besoins, dans le développement des tissus et la formation du sang ${ }^{36}$. Moreau confirme cette hypothèse chez vingt-cinq nouveaux-nés. De plus, il détermine le rapport du fer hémoglobinique sur le fer total de l'organisme, un paramètre dont il étudie les variations dans divers états pathologiques.

Professeur adjoint d'Hugounenq dans la chaire de "Chimie biologique et médicale », Étienne Barral (1860-1938) conduit avec le médecin Raphaël Lépine (1840-1919) des travaux

\footnotetext{
${ }^{31}$ Anonyme (1890), pp. 79.

${ }^{32}$ Moreau B. (1922), Op. cit., p. 9

${ }^{33}$ Ce puceron parasite, qui ravage le vignoble français depuis les années 1860 , se trouve à l'origine d'une crise agricole sans précédent. Cf. Pouget R. (1990) Histoire de la lutte contre le Phylloxera de la vigne en France, Paris, INRA, $157 \mathrm{p}$.

${ }^{34}$ Moreau B. (1903), Op. cit., p. 90-91.

35 Hugounenq L. (1934) "Notice nécrologique sur M. P. Cazeneuve, Associé national, Bulletin de l'Académie nationale de Médecine, T. CXI, n²2, p. 832.

${ }^{36}$ Bretin P. (1924), Op. cit., p. 109
} 
pionniers sur la glycémie et sa régulation. Les deux chercheurs font une découverte importante dans le contexte suivant: Paul Langerhans (1847-1888) décrit en 1869 ses fameux «îlots » pancréatiques, Apollinaire Bouchardat (1806-1886) observe en 1870 une amélioration de ses patients diabétiques en état de sous-nutrition et la distinction entre diabète gras et maigre se trouve établie en 1880 par Étienne Lancereaux (1829-1910). Neuf ans plus tard, Barral et Lépine démontrent que «le pancréas cède au sang un ferment qui contribue à la destruction du sucre et que l'on peut nommer : ferment glycolytique $\gg{ }^{37} \mathrm{Ce}$ dernier se forme dans la glande sans s'y accumuler, comme l'observent ultérieurement les deux chercheurs: une voie s'ouvre, qui conduira à l'isolement de l'insuline.

Albert Morel (1875-1953) - qui succèdera à Cazeneuve dans la chaire de «Chimie organique et toxicologie »- devient docteur en médecine grâce à des Recherches physiologiques sur l'utilisation des graisses du sang (1902). Dans le domaine de la chimie appliquée à la physiologie et à la pathologie, il réalise divers travaux liés au sang : effets des augmentations de pression, étude de la coagulation, des variations pathologiques de la teneur en albumines sériques, mise en évidence de combinaisons chloruro-protéiques, de l'urobiline ou de la choline ${ }^{38}$.

L'hématologie médico-légale se trouve brillamment illustrée par les travaux de Florence. Cet élève de Lacassagne ${ }^{39}$ soutient sa thèse de doctorat en médecine sur Les taches de sang, leur signification, leur importance en médecine judiciaire (1884). Florence ne quittera plus, désormais, la voie de l'hématologie médico-légale. Afin de détecter ce qu'il nomme les « taches critiques »- petites, altérées, dispersées dans la texture des supports poreux -, il met au point un réactif sensible portant son nom. Florence collabore avec la firme Nachet, concevant de nouvelles techniques instrumentales adaptées au sang ${ }^{40}$. Aux côtés des frères Lumière, le savant signe des autochromes obtenus à partir de globules rouges d'oiseaux extraits de fibres textiles. Florence met également au point des réactions permettant de détecter le sang et l'urobiline dans les urines, ou bien les taches de sperme ${ }^{41}$. Ces derniers travaux entrent en résonance avec deux notes de Cauvet sur les taches de sang et de sperme, publiées en $1875^{42}$.

L'œuvre d'Hugounenq apparaît, à première vue, comme très diversifiée : découverte de l'acide $\beta$-oxybutyrique lévogyre dans le sang d'un diabétique ${ }^{43}$, effets des constituants du vin et des colorants de la houille sur la digestion peptique ${ }^{44}$, action des radiations lumineuses sur les

\footnotetext{
${ }^{37}$ Desgrez P. (1992) “Le professeur Étienne Barral (1860-1936)”, Revue d’Histoire de la Pharmacie, n²94, pp. 325-327.

${ }^{38}$ Chambon M. (1953), "Professeur Albert Morel (1875-1953)”, Annales Pharmaceutiques Françaises, vol. 11, n³, pp. 238-241.

${ }^{39}$ Il est également élève de Élève de Cazeneuve et Crolas.

${ }^{40}$ Il s'agit de la mise au point d'une technique d'analyse spectroscopique du sang solidifié et de la construction d'un microscope à éclairage interne - permettant de détecter les globules rouges sur des aiguilles ou des lames métalliques.

${ }^{41}$ Morel A. (1927) Le professeur Albert Florence - 1851-1927, Bulletin des Sciences Pharmacologiques, vol. 34, n 12 , pp. 706-707.

${ }^{42}$ Ces articles relatent deux expertises, diligentées à la suite de suspicions de viol ou d'attentat à la pudeur. Cf. Cauvet D. (1875) "Taches de sperme et taches de sang", Annales d'Hygiène Publique et de Médecine Légale, vol. 44, pp. 117-126 et Cauvet D. (1875) “Attentat à la pudeur”, Id., pp. 367-373.

${ }^{43}$ Cf. Hugounenq L. (1890) Exposé des titres scientifiques, Lyon, Association typographique, p. 16 et Weitz R. (1942) Op. cit., p. 91.

${ }^{44}$ Il s'agit d'investigations conduites dans le cadre de sa thèse de pharmacie, Cf. Hugounenq L. (1891) Recherches nouvelles sur les vins, Lyon, Storck, 32 p.
} 
stérols, biochimie des électrolytes, etc ${ }^{45}$. Sans oublier des recherches effectuées en collaboration avec le physiologiste Doyon ${ }^{46}$. Mais, un axe thématique majeur traverse l'oeuvre du pharmacien : il s'agit de la biochimie des molécules azotées. Hugounenq étudie au début des années 1880 la production physiologique de l'urée, pour laquelle il met au point des techniques de dosage. Puis Les alcaloïdes d'origine animale, leucomaïnes et ptomaïnes, retiennent son attention. Il leur consacre sa thèse d'agrégation, publiée en 1886, et conclut à «l'identité des phénomènes qui donnent naissance à la formation des alcaloïdes dans les cellules animales et végétales ${ }^{47}$. Ultérieurement, cette conception se trouvera infirmée et, comme les leucomaïnes, les ptomaïnes ne seront plus classées dans le groupe des alcaloïdes ${ }^{48}$. Au début du XXème siècle, après avoir visité les grands laboratoires allemands, Hugounenq décide de se lancer dans l'étude des protéines. Il confirme l'enchainement peptidique de leur structure primaire - postulé par Emil Fischer (1852-1919) -, avant de réaliser des expériences d'hydrolyse systématique. Hugounenq émet ensuite des hypothèses sur plusieurs phénomènes, comme les relations existant entre les enzymes et les minéraux, ou entre la nature des protéines et leur cinétique d'hydrolyse, ou encore entre le coma diabétique et une accumulation de polypeptides dans le plasma. Enfin, le savant met au point une méthode de synthèse de cyclopeptides in vitro, à partir d'aminoacides ${ }^{49}$.

Morel collabore avec Hugounenq sur diverses questions de chimie biologique : la constitution des matières albuminoïdes, l'origine de l'hémoglobine, la digestion enzymatique des gommes, le dosage des albumines et de l'urée retiennent notamment son attention ${ }^{50}$.

\section{4) - Pharmacologie et toxicologie}

Le domaine de la pharmacologie sensu stricto - étude du devenir et des effets des médicaments introduits dans l'organisme - est faiblement représenté dans les travaux des professeurs de la faculté mixte. Il faut dire que le temps des investigations sur des organes isolés, des cultures de cellules ou des fractions subcellulaires n'est pas encore venu, encore moins celui des modélisations mathématiques pharmacocinétiques - annoncées cependant par Moreau (cf. infra). Un seul exemple pharmacologique sera fourni ici. Étudiant en 1889 les effets de l'antipyrine, Crolas et Hugounenq concluent «qu'à l'état normal [le principe actif] n'a pas d'action sur le sang et l'excrétion urinaire ; c'est un médicament du système nerveux $»^{51}$.

A contrario, diverses branches de la toxicologie sont explorées: toxicologie végétale, toxicologie des polluants ou des produits de synthèse, toxicologie médicamenteuse, toxicologie médico-légale, etc.

En toxicologie végétale, Beauvisage est l'auteur d'un petit ouvrage sur la nocivité des graines de ricin ${ }^{52}$. Bretin étudie l'action irritante pour la peau de certaines plantes, dans sa thèse

\footnotetext{
${ }^{45}$ Morel A. (1943) "L'œuvre scientifique du chimiste Louis Hugounenq", Journal de Médecine de Lyon, 5 mars 1943, pp. 149-151.

${ }^{46}$ D'où des publications conjointes, sur des sujets comme les pigments biliaires, le traitement du diabète par des extraits de pancréas ou l'action chimique de certaines bactéries.

${ }^{47}$ Hugounenq L. (1890), Op. cit., p. 15.

${ }^{48}$ Mais, de véritables alcaloïdes animaux seront découverts à la fin du XXème siècle.

${ }^{49}$ Morel A. (1943), Op. cit., p. 150.

${ }^{50}$ Chambon M. (1953), "Professeur Albert Morel (1875-1953)", Annales Pharmaceutiques Françaises, vol. 11, n³, pp. 238-241.

${ }^{51}$ Hugounenq L. (1890) Op. cit., p. 17.

${ }^{52}$ Beauvisage G. (1894) Toxicité des graines de ricin, Paris, J-B. Baillière \& fils, 31 p.
} 
de doctorat en médecine intitulée De l'origine végétale de certaines dermatites (1909). « Près de deux-cents espèces de plantes appartenant à quarante-deux familles botaniques sont signalées comme susceptibles de produire des dermatites allant de la rubéfaction simple jusqu'aux pustules d'ecthyma et aux furoncles $»^{53}$. En 1889, Cazeneuve et Hugounenq signalent à leur collègue Barral l'existence d'empoisonnements liés à des adultérations d'anis étoilé par des «badianes toxiques ». Barral conduit alors une étude toxicologique complète sur l'une des plantes incriminées, Illicium parviflorum. Chez le Chien, il détermine les quantités de poudre végétale dangereuse et observe les symptômes de l'empoisonnement. Il localise dans le fruit de la plante le principe toxique, un hétéroside qu'il isole et baptise parvillicine ${ }^{54}$.

Dans le domaine de la toxicologie chimique, le monoxyde de carbone occupe une place importante aux débuts de la faculté mixte lyonnaise. Les accidents redoutables qu'il entraine sont liés, le plus souvent, à des dysfonctionnements de dispositifs de chauffage. Ainsi, Moreau diriget-il, dans son laboratoire, une thèse de doctorat en pharmacie intitulée Contribution à l'étude de la toxicité des produits de combustion de quelques appareils de chauffage et d'éclairage au gaz. $(1906)^{55}$. Sa formation de physico-chimiste conduit Sambuc ${ }^{56}$ à modéliser l'organisme selon la règle des phases de Willard Gibbs, pour comprendre les phénomènes chimiques liés à l'intoxication oxycarbonée. Il réduit ainsi le corps humain et son milieu à un « système formé de trois composants indépendants (oxygène, oxyde de carbone, hémoglobine réduite), et partagé en quatre phases, dont trois solides (hémoglobine réduite, oxyhémoglobine, carboxyhémoglobine) et une gazeuse »- le mélange d'oxygène et d'oxyde de carbone ${ }^{57}$. Ce modèle permet à Sambuc d'expliquer la pathogénie de l'intoxication, en accord avec les études précédemment réalisées par Claude Bernard. L'étude du pharmacien est éditée en 1904 par les Archives d'Anthropologie criminelle et de Criminologie ${ }^{58}$. L'année suivante, dans le même périodique, Sambuc publie des considérations sur l'intoxication aigüe par le phosphore. Comme pour l'oxyde de carbone, il recherche une explication physico-chimique de phénomènes biologiques. Sambuc rapproche, de manière originale, le tableau clinique de l'intoxication phosphorée de celui observée chez les malades atteints de la fièvre jaune ${ }^{59}$.

Au cours de sa démarche scientifique et politique de protection de l'hygiène publique - il est membre du Conseil d'hygiène départemental du Rhône -, Cazeneuve aborde le champ de la toxicologie. La catastrophe du Phylloxéra (cf. supra) suscite de nombreuses opérations frauduleuses, visant à améliorer les caractères organoleptiques de mauvais vins. En particulier, des matières colorantes dérivées du goudron de houille - tels la fuschine et les dérivés azoïques sont utilisés comme additifs. Cazeneuve se préoccupe de l'éventuelle toxicité de ces substances pour les consommateurs. Il publie trois ouvrages sur la question, faisant état dans le dernier d'entre eux ${ }^{60}$ d'expériences réalisées avec le vétérinaire Saturnin Arloing (1846-1911) et Lépine :

\footnotetext{
${ }^{53}$ Moreau B. (1922), Op. cit., pp. 7-8.

${ }^{54}$ Barral E. (1910) Exposé des titres et travaux scientifiques, Lyon, Rey, pp. 60-65.

${ }^{55}$ Bretin P. (1924), Op. cit., p. 110

${ }^{56}$ De 1910 à 1912, Sambuc dispense un cours de toxicologie à la faculté mixte de Lyon.

${ }^{57}$ Sambuc C. (1913) Op. cit., p.22.

${ }^{58}$ Sambuc C. (1904) "Sur les conditions de l'empoisonnement par l'oxyde de carbone", Archives d'Anthropologie criminelle et de Criminologie, nouvelle série, T. III, p. 241 et Sambuc C. (1913) Op. cit., p. 22-24.

59 Sambuc C. (1905) «Revue critique des travaux relatifs à l'intoxication phosphorée aigüe » Archives d'Anthropologie criminelle et de Criminologie, T. V, p. 299 et Sambuc C. (1913) Op. cit., p. 25-26

${ }^{60}$ Cazeneuve P. (1887) Les colorants de la houille au point de vue toxicologique et hygiénique, Lyon, Association typographique, $169 \mathrm{p}$.
} 
celles-ci démontrent l'absence de nocivité des colorants généralement retrouvés dans les vins. Cazeneuve alerte l'Académie de médecine Sur les dangers de l'emploi des insecticides à base arsenicale en agriculture (1908) et publie des articles sur la réglementation des substances vénéneuses et les empoisonnements alimentaires ${ }^{61}$.

En raison des compétences toxicologiques de Barral, son expertise est sollicitée en 1926 dans l'affaire Bougrat ${ }^{62}$. Ce médecin marseillais est inculpé d'homicide sur l'un de ses patients, décédé à la suite d'une injection de norvarsénobenzole. Chargé d'effectuer une analyse toxicologique sur les viscères du cadavre, Barral conclut à la présence de quantités d'arsenic et de mercure insuffisantes pour entrainer la mort. Ses conclusions sont confirmées par un second expert : Alexandre Desgrez, professeur à la faculté de médecine de Paris ${ }^{63}$.

Travaillant avec Lacassagne, Hugounenq s'intéresse à la toxicologie du cyanure de potassium. Il note que l'empoisonnement entraine l'accumulation, dans les premières parties de l'intestin, d'ammoniaque susceptible d'interférer avec les réactions chimiques caractéristiques du cyanure $^{64}$.

\section{Conclusion}

Les activités scientifiques des premiers professeurs de pharmacie de la faculté mixte lyonnaise sont nombreuses et importantes, dans le domaine des sciences de la vie et de la santé. Elles transcendent les découpages disciplinaires qu'imposent les chaires magistrales. Ainsi, des botanistes comme Cauvet et Bretin, ou des chimistes organiciens comme Sambuc ou Moreau, n'hésitent pas à s'impliquer dans des travaux relevant de la médecine légale.

Les recherches qui viennent d'être décrites s'associent, plus ou moins directement, à des travaux d'enseignement ou de vulgarisation scientifique. Ainsi, outre la conservation d'un droguier, la chaire de "Matière médicale et botanique» se trouve en charge d'un jardin botanique «pédagogique» de $3000 \mathrm{~m}^{2}$. L'organisation de ses plantations, conçue par Beauvisage $^{65}$ selon les principes de la «méthode naturelle » de Bernard (1699-1777) et AntoineLaurent de Jussieu (1746-1836), constitue un véritable «développement en surface» de la taxinomie végétale ${ }^{66}$. En 1889, Beauvisage publie un guide pédagogique du jardin ${ }^{67}$, lequel sera ensuite régulièrement réédité en collaboration avec Bretin. Bien d'autres ouvrages destinés aux étudiants, qu'il s'agisse de traités ou de précis, d'histoire naturelle, de matière médicale, de chimie biologique ou de toxicologie, sont édités par les professeurs de pharmacie de la faculté mixte : Cauvet, Barral, Hugounenq, Morel, etc.

\footnotetext{
${ }^{61}$ Chambon M. (1934), Op. cit., pp. 360-361.

${ }^{62}$ Cette affaire a eu un grand retentissement dans le milieu médical et auprès du grand public. Cf. Mure A. (1954) La piqûre mortelle, Lyon, Paris, La Flamme d'Or, 127 p. et Dedet C. (2000) Le secret du Dr. Bougrat - Marseille Cayenne - Caracas, l'aventure d'un proscrit, Paris, Phébus, 467 p.

63 Desgrez P. (1992) Op. cit., pp. 327-328.

${ }^{64}$ Hugounenq L. (1890) Op. cit., p. 19-20.

65 Beauvisage conçoit, dès sa création en 1888, les plans du jardin dont il est nommé officiellement directeur en 1898. Il conserve jusqu'en 1909 cette fonction, que ses collègues et successeurs de la chaire de "Matière médicale et Botanique" assumeront ensuite.

${ }^{66}$ Beauvisage G. (1891) Op. cit.p. 17

${ }^{67}$ Beauvisage G. (1889) Guide des étudiants en médcine et en pharmacie et des élèves herboristes au jardin botanique de la faculté de Lyon (avec un plan du jardin), Lyon, Librairie Générale Henri Georg.
} 
Quant à la diffusion des connaissances scientifiques vers le grand public, elle se trouve bien illustrée par les nombreux articles que publient, dans la presse nationale, Cazeneuve - sur l'hygiène et la protection de la santé publique - et Beauvisage - sur l'enseignement et la scolarisation des enfants handicapés.

Le souvenir de plusieurs universitaires qui viennent d'être évoqués plane encore sur le quartier où se dresse l'actuelle faculté de pharmacie de Lyon. En 1930, celle-ci - toujours associée à la médecine - quitte le palais Hirsch et les quais du Rhône pour le quartier GrangeBlanche. Le jardin botanique, transplanté à proximité des nouveaux bâtiments, construits grâce à l'aide de la fondation Rockefeller, «n'a rien perdu de son éclat». Encore aujourd'hui, le promeneur circulant autour du «campus Rockefeller» peut emprunter des rues aux noms évocateurs : Beauvisage, Hugounenq, Cazeneuve. Il peut également faire sienne une citation d'Euripide, appliquée à Hugounenq par l'un de ses biographes : « " Heureux qui possède la science! Il ne cherche pas à usurper sur ses concitoyens, il ne médite pas d'action injuste. Contemplant la nature éternelle, l'ordre inaltérable, l'origine et les éléments des choses, son âme n'est ternie d'aucun désir honteux ${ }^{68}$.

${ }^{68}$ Florence G. (1943) "La pensée scientifique de Louis Hugounenq", Journal de Médecine de Lyon, 5 mars 1943, p. 148. 


\section{RÉFÉRENCES}

Anonyme (1890) “Mr. Le Professeur Cauvet”, L’Union Pharmaceutique, 31ème année, pp. 77-81.

Barral E. (1910) Exposé des titres et travaux scientifiques, Lyon, Rey, 65 p.

Beauvisage G. (1883) Les galles utiles, Paris, Doin, 99 p.

Beauvisage G. (1889) Guide des étudiants en médcine et en pharmacie et des élèves herboristes au jardin botanique de la faculté de Lyon (avec un plan du jardin), Lyon, Librairie Générale Henri Georg, 75 p.

Beauvisage G. (1891) Exposé des titres et travaux scientifiques, Lyon, Association typographique, 24 p.

Beauvisage G. (1894) Toxicité des graines de ricin, Paris, J-B. Baillière \& fils, 31 p.

Bretin P. (1924) "Notice biographique. Le Professeur Moreau, Bulletin des Sciences Pharmacologiques, T. 31, n², pp. 105-112.

Cauvet D. (1875) “Taches de sperme et taches de sang”, Annales d'Hygiène Publique et de Médecine Légale, vol. 44, pp. 117-126.

Cauvet D. (1875) “Attentat à la pudeur, Annales d'Hygiène Publique et de Médecine Légale, vol. 44, pp. 367-373.

Chambon M. (1934) "Le professeur Paul Cazeneuve (1852-1934)", Bulletin des Sciences Pharmacologiques, T. 41, n6, p. 357-367.

Chambon M. (1953), "Professeur Albert Morel (1875-1953)”, Annales Pharmaceutiques Françaises, vol. 11, n³, pp. 238-241.

Desgrez P. (1992) “Le professeur Étienne Barral (1860-1936)”, Revue d'Histoire de la Pharmacie, n²94, p. 323329.

Despierres G. (1987) «L'École secondaire de Médecine (1821-1841), in : Bouchet A. La médecine à Lyon - Des origines à nos jours, Lyon, Hervas, pp. $92-95$ et Despierres G. (1987) « L'École préparatoire de Médecine et de Pharmacie », Ibid, pp. $96-100$.

Despierres G. (1987) «La Faculté mixte de Médecine et de Pharmacie et son éclatement», in: Bouchet A. La médecine à Lyon - Des origines à nos jours, Lyon, Hervas, pp. 101- 116 et Cazeneuve P. (1920) Sur les origines de la faculté de médecine et de pharmacie de Lyon, Lyon, Association typographique.

Drevon B. (1977) «La pharmacie dans la faculté mixte de médecine et pharmacie de Lyon », in: Le Centenaire de la faculté mixte de médecine et de pharmacie de Lyon, Cahiers de médecine, vol. 2, n³6, pp. 2067-2069.

Guiart J. (1941) L'école médicale lyonnaise : catalogue commenté de la section régionale du musée historique de la faculté mixte de médecine et pharmacie de Lyon, Paris, Masson, pp. 106-107.

Florence G. (1943) "La pensée scientifique de Louis Hugounenq", Journal de Médecine de Lyon, 5 mars 1943, pp. 147-148.

Hugounenq L. (1890) Exposé des titres scientifiques, Lyon, Association typographique, 23 p.

Hugounenq L. (1891) Recherches nouvelles sur les vins, Lyon, Storck, 32 p.

Hugounenq L. (1934) "Notice nécrologique sur M. P. Cazeneuve, Associé national, Bulletin de l'Académie nationale de Médecine, T. CXI, n²2, p. 832-833.

Labrude P. (2009) "Nouvelles recherches sur Philippe Désiré Cauvet (Agde 1827-Lyon 1890), pharmacien militaire, naturaliste, agrégé et professeur, Revue d’Histoire de la Pharmacie, n³64, pp. 385-398.

Manceau P. (1931) "Le professeur Philippe Bretin (1874-1931)”, Lyon Pharmaceutique, pp. 57-65.

Moreau B. (1903) "Le professeur Crolas", Bulletin des Sciences Pharmacologiques, T. 8, p. 82-92.

Moreau B; (1908) "Henri-Eugène Causse", Bulletin des Sciences Pharmacologiques, T. 15, pp. 114-116.

Moreau B. (1922) Rapport sur la candidature de Monsieur le Docteur Bretin, Agrégé, Chargé de Cours, à la chaire de Matière médicale et Botanique de la Faculté de Médecine et Pharmacie de Lyon. Document manuscrit. Dossier personnel de Philippe Bretin, ISPB - Faculté de Pharmacie de Lyon, Université Claude Bernard Lyon 1, p. 9.

Morel A. (1927) "Le professeur Albert Florence - 1851-1927", Bulletin des Sciences Pharmacologiques, vol. 34, $\mathrm{n}^{\circ} 12$, p. 703-708.

Morel A. (1943) “L'œuvre scientifique du chimiste Louis Hugounenq", Journal de Médecine de Lyon, 5 mars 1943, pp. 149-151.

Paris R. (1954) «Botanique médicale », in : Davy de Virville, A., Histoire de la botanique en France, Paris, Société d'Édition d'Enseignement Supérieur, pp. 301-306.

Pouget R. (1990) Histoire de la lutte contre le Phylloxera de la vigne en France, Paris, INRA, 157 p.

Sambuc C. (1913) Titres et services du Dr Camille Sambuc, Lyon, Rey, 29 p.

Weitz R. "Le doyen honoraire Louis-Joseph Hugounenq (1860-1942)", Bulletin des Sciences Pharmacologiques, vol. $49, \mathrm{n}^{\circ} 10-11-12$, p. 91. 
